\title{
Epidemiology of Acute Kidney Injury in Critically III Children and Young Adults
}

\author{
Ahmad Kaddourah, M.D., Rajit K. Basu, M.D., Sean M. Bagshaw, M.D., Stuart L. Goldstein, \\ M.D., and for the AWARE Investigators ${ }^{\star}$ \\ Center for Acute Care Nephrology (A.K., R.K.B., S.L.G.) and the Division of Critical Care (R.K.B.), \\ Cincinnati Children's Hospital Medical Center, Cincinnati; Sidra Medical and Research Center, \\ Doha, Qatar (A.K.); and the Department of Critical Care Medicine, Faculty of Medicine and \\ Dentistry, University of Alberta, Edmonton, Canada (S.M.B.).
}

\begin{abstract}
Background-The epidemiologic characteristics of children and young adults with acute kidney injury have been described in single-center and retrospective studies. We conducted a multinational, prospective study involving patients admitted to pediatric intensive care units to define the incremental risk of death and complications associated with severe acute kidney injury.

Methods-We used the Kidney Disease: Improving Global Outcomes criteria to define acute kidney injury. Severe acute kidney injury was defined as stage 2 or 3 acute kidney injury (plasma creatinine level $\geq 2$ times the baseline level or urine output $<0.5 \mathrm{ml}$ per kilogram of body weight per hour for $\geq 12$ hours) and was assessed for the first 7 days of intensive care. All patients 3 months to 25 years of age who were admitted to 1 of 32 participating units were screened during 3 consecutive months. The primary outcome was 28 -day mortality.
\end{abstract}

Results-A total of 4683 patients were evaluated; acute kidney injury developed in 1261 patients (26.9\%; 95\% confidence interval [CI], 25.6 to 28.2 ), and severe acute kidney injury developed in 543 patients (11.6\%; 95\% CI, 10.7 to 12.5$)$. Severe acute kidney injury conferred an increased risk of death by day 28 after adjustment for 16 covariates (adjusted odds ratio, 1.77; 95\% CI, 1.17 to 2.68); death occurred in 60 of the 543 patients (11.0\%) with severe acute kidney injury versus 105 of the 4140 patients $(2.5 \%)$ without severe acute kidney injury $(\mathrm{P}<0.001)$. Severe acute kidney injury was associated with increased use of mechanical ventilation and renal-replacement therapy. A stepwise increase in 28-day mortality was associated with worsening severity of acute kidney injury ( $\mathrm{P}<0.001$ by log-rank test). Assessment of acute kidney injury according to the plasma creatinine level alone failed to identify acute kidney injury in $67.2 \%$ of the patients with low urine output.

Conclusions-Acute kidney injury is common and is associated with poor outcomes, including increased mortality, among critically ill children and young adults. (Funded by the Pe-diatric

\footnotetext{
*A complete list of investigators in the Assessment of Worldwide Acute Kidney Injury, Renal Angina, and Epidemiology (AWARE) study is provided in the Supplementary Appendix, available at NEJM.org.

Address reprint requests to Dr. Goldstein at the Center for Acute Care Nephrology, Cincinnati Children's Hospital Medical Center, 3333 Burnet Ave., MLC 7022, Cincinnati, OH 45229, or at stuart.goldstein@ cchmc.org.

Disclosure forms provided by the authors are available with the full text of this article at NEJM.org.
} 
Nephrology Center of Excellence at Cincinnati Children's Hospital Medical Center and others; AWARE ClinicalTrials.gov number, NCT01987921.)

Epidemiologic studies involving adults have shown that acute kidney injury is associated with increased mortality, prolonged mechanical ventilation, and prolonged length of stay in intensive care units (ICUs). ${ }^{1-3}$ A multinational, prospective study involving 1802 adults ${ }^{3}$ initiated the use of Kidney Disease: Improving Global Outcomes (KDIGO) guidelines to describe the epidemiology of acute kidney injury; the guidelines ${ }^{4}$ define and stage acute kidney injury according to the plasma creatinine level and urine output (Table S1 in the Supplementary Appendix, available with the full text of this article at NEJM.org). That study showed graded associations between the severity of acute kidney injury and outcomes. A single-center study involving adults revealed that mortality and morbidity were higher when both the plasma creatinine level and urine output were used to diagnose acute kidney injury than when either was used alone..$^{5}$ In comparison, the study of acute kidney injury in children has been limited. ${ }^{6-9}$ Four single-center, retrospective studies involving children have used the KDIGO criteria, ${ }^{10-13}$ but none of those assessed urine output in the diagnosis of acute kidney injury.

In studies involving adults, chronic coexisting conditions (e.g., diabetes and cirrhosis) commonly confound the interpretation of the risk that acute kidney injury confers with respect to mortality. Children generally do not have chronic coexisting conditions, so pediatric studies provide a unique opportunity to show a direct role of acute kidney injury in mediating an increased risk of death.

We conducted the Assessment of Worldwide Acute Kidney Injury, Renal Angina, and Epidemiology (AWARE) study ${ }^{14}$ to define the epidemiology of acute kidney injury and to characterize risk factors for acute kidney injury and associated morbidity in a multinational cohort of critically ill children and young adults. We hypothesized that severe acute kidney injury was common and would increase the risk of death.

\section{Methods}

\section{Study Design and Cohort}

This was a prospective, observational study that recruited patients in 32 pediatric ICUs across Asia, Australia, Europe, and North America for 3 consecutive months in 2014; the design has been published previously. ${ }^{14}$ In brief, all patients between 3 months and 25 years of age with a predicted ICU stay of at least 48 hours were eligible. Exclusion criteria were an estimated glomerular filtration rate (GFR) below $15 \mathrm{ml}$ per minute per $1.73 \mathrm{~m}^{2}$ of bodysurface area, maintenance dialysis, or receipt of a kidney transplant in the preceding 90 days. We reviewed the medical records of eligible patients to collect data for the following time periods: up to 3 months before admission (plasma creatinine level only), daily during the first 7 days after ICU admission, and on day 28 after admission. Data on 28-day outcomes were recorded regardless of whether the patient was still in the ICU or had been discharged or had died. All centers obtained approval from health research ethics boards before commencement of the study. 


\section{Definitions and Measurements}

The baseline plasma creatinine level was defined as the lowest level in the 3 months before admission. The estimated GFR was calculated with the use of the original Schwartz formula. ${ }^{15}$ When the baseline plasma creatinine level was unavailable, the estimated GFR was assumed to be $120 \mathrm{ml}$ per minute per $1.73 \mathrm{~m}^{2}$, as validated previously. ${ }^{16} \mathrm{We}$ applied KDIGO criteria for the plasma creatinine level and urine output to define and classify acute kidney injury; however, use of renal-replacement therapy was a secondary outcome and was omitted from the definition of stage 3 kidney injury. ${ }^{4}$ We required at least one plasma creatinine measurement or at least 12 hours of recorded urine output in the first 7 days after admission to assess for acute kidney injury. When the two criteria resulted in different stages, we chose the higher stage. We defined severe acute kidney injury as stage 2 or 3 acute kidney injury (plasma creatinine level $\geq 2$ times the baseline level or urine output $<0.5$ $\mathrm{ml}$ per kilogram of body weight per hour for $\geq 12$ hours), because these stages have been associated with increased mortality in studies involving children. ${ }^{7,11,17}$ To evaluate the effect of the severity of acute kidney injury on secondary outcomes, we defined the maximum stage of acute kidney injury as the highest stage observed during the first 7 days after ICU admission.

\section{Outcomes}

The primary outcome was 28-day mortality. Secondary outcomes were length of stay in the ICU, receipt and duration of mechanical ventilation, receipt of extracorporeal membrane oxygenation, and receipt of renal-replacement therapy.

\section{Statistical Analysis}

Categorical data are presented as counts and percentages and were analyzed with the chisquare test and Fisher's exact test, as appropriate. Normality was tested with the Kolmogorov-Smirnov test. Skewed distributions are described with medians and interquartile ranges and were compared with the use of the Wilcoxon rank-sum test and Kruskal-Wallis test. Normal distributions are described with means and standard deviations and were compared with the use of Student's t-test. The two-sided alpha level was set at 0.05 . With respect to missing data, we performed sensitivity analyses to assess for differences in demographic and clinical characteristics, the rate of acute kidney injury, and secondary outcomes when data on the 28-day outcome (alive vs. dead) were unavailable and to assess for differences in 28-day mortality and secondary outcomes when data on the baseline plasma creatinine level were unavailable (Tables S2 and S3 in the Supplementary Appendix).

We used multivariable logistic-regression models for the primary analysis to determine the association between the maximum stage of acute kidney injury and risk of death, with adjustment for risk factors that differed between survivors and nonsurvivors. Variables were entered into models when the alpha level of the risk factor was less than 0.15 in bivariate analysis. Kaplan- Meier survival curves and log-rank tests were used to describe the effect of the maximum stage of acute kidney injury on 28-day mortality. The preferred illnessseverity scoring system at each center (the Pediatric Risk of Mortality III [PRISM-III] score, Pediatric Logistic Organ Dysfunction [PELOD] score, or Pediatric Index of Mortality 2 
[PIM-2] score $)^{18}$ was incorporated in the model for the patients at that center. The risk of death associated with acute kidney injury was evaluated as a categorical variable. In this multivariate analysis, a maximum stage of 2 or 3 was considered to be severe acute kidney injury, and no acute kidney injury or stage 1 was the reference. Linear regression models were created to determine the predictors of length of stay in the ICU. Analyses were conducted with the use of JMP software, version 11.0.0 (SAS Institute), and Microsoft Excel 2010. The protocol and statistical analysis plan are available at NEJM.org.

\section{Results}

\section{Patient Characteristics}

We screened 6821 patients, of whom 5237 (76.8\%) met the eligibility criteria. Data on 28day outcomes were available for 4984 patients (95.2\%). Figure 1 shows the process from screening to assessment of the primary outcome. Patient demographic and clinical characteristics are shown in Table 1.

A total of 4683 patients had known status with respect to both acute kidney injury and 28day outcome; among these patients, acute kidney injury developed in 1261 patients (26.9\%; 95\% confidence interval [CI], 25.6 to 28.2) and severe acute kidney injury developed in 543 (11.6\%; $95 \%$ CI, 10.7 to 12.5$)$ during the first 7 days after ICU admission. The maximum stage of acute kidney injury was stage 1 in 718 patients (15.3\%), stage 2 in 294 patients (6.3\%), and stage 3 in 249 patients (5.3\%).

Of the 4984 patients with data on 28-day outcomes, 73 (1.5\%; 95\% CI, 1.2 to 1.8$)$ received renal-replacement therapy. A total of 169 patients $(3.4 \%$; 95\% CI, 2.9 to 3.9$)$ died within 28 days (148 in the ICU and 21 after discharge from the ICU). Nearly all clinical factors were similar in patients with available data on mortality and the 253 patients with missing data on mortality, and mortality was identical when we included these 253 patients after estimating their mortality on the basis of their identified risk factors for death (Table S2 in the Supplementary Appendix). The 2533 patients without an available measured baseline plasma creatinine level had shorter stays in the ICU and lower use of renal-replacement therapy and mechanical ventilation than the 2451 patients for whom a baseline plasma creatinine level was available. No such associations were seen in the subgroup of 102 patients with a known renal or urologic coexisting condition; within that subgroup, there were no significant differences in these variables between those who did not have an available measured baseline plasma creatinine level and those who did (Table S3A and S3B in the Supplementary Appendix).

\section{Severe Acute Kidney Injury as an Independent Risk Factor for Death}

Comparative analyses between survivors and non-survivors are shown in Table 2. On average, the maximum stage of acute kidney injury was higher in nonsurvivors than in survivors. Severe acute kidney injury was associated with an increased risk of death (odds ratio, $1.77 ; 95 \%$ CI, 1.17 to 2.68) after adjustment for 16 covariates. Death occurred in 60 of the 543 patients $(11.0 \%)$ with severe acute kidney injury as compared with 105 of the 4140 patients $(2.5 \%)$ without severe acute kidney injury $(\mathrm{P}<0.001)$. The use of renal-replacement 
therapy was the second strongest predictor (after the use of vasoactive support) of death by day 28 after admission (odds ratio, 3.38; 95\% CI, 1.74 to 6.54). Severe acute kidney injury conferred an increased risk of death after adjustment for center-preferred illness-severity scores ( $\mathrm{P}<0.001$ for each score: PRISM-III [1263 patients], PIM-2 [1363 patients] and PELOD [859 patients]), with odds ratios ranging from 2.41 to 5.12 ( $\mathrm{P}<0.05$ across scores) (Table S4A in the Supplementary Appendix).

\section{Maximum Stage of Acute Kidney Injury and Outcomes}

A stepwise increase in the maximum stage of acute kidney injury conferred an incremental risk of death $(\mathrm{P}<0.001)$ (Fig. 2). The risk of death associated with a maximum stage of 3 exceeded the corresponding risk associated with no acute kidney injury or a maximum stage of 1 or 2 ( $\mathrm{P}<0.001$ for all comparisons). A maximum stage of 2 carried increased risk when compared with no acute kidney injury but not when compared with a maximum stage of 1 . Patients with a maximum stage of 1 had longer ICU stays and greater provision and duration of mechanical ventilation than patients without acute kidney injury. Increases in the maximum stage of acute kidney injury were associated with increased length of stay after adjustment for illness-severity scores (Table S4B in the Supplementary Appendix). An increase in the maximum stage of acute kidney injury was associated with increased use of renal-replacement therapy, increased use of extracorporeal membrane oxygenation, and increased use and duration of mechanical ventilation (Table S5 in the Supplementary Appendix).

\section{Comparison of Plasma Creatinine Level and Urine Output in Defining Acute Kidney Injury}

Data on the plasma creatinine level (4036 patients) or urine output (3965 patients) were available to assess for acute kidney injury in $94 \%$ of the patients with known 28-day outcomes; 3318 patients had data on both. Mortality was higher among patients with stage 3 acute kidney injury defined according to urine output than among those with stage 3 acute kidney injury defined according to the plasma creatinine level (Fig. S1 in the Supplementary Appendix).

In patients with data on both the plasma creatinine level and urine output, acute kidney injury defined according to the plasma creatinine level developed in 738 patients $(22.2 \%)$ and acute kidney injury defined according to urine output developed in 528 patients (15.9\%). In 355 of the 528 patients (67.2\%) who met the urine-output criteria for acute kidney injury, the diagnosis of acute kidney injury would have been missed if only the plasma creatinine criteria had been used. Mortality was higher among patients with low urine output than among those with normal urine output $(7.8 \%$ vs. $2.9 \%, \mathrm{P}=0.02)$.

\section{Progression of Acute Kidney Injury}

The daily prevalence of acute kidney injury increased progressively from $14.5 \%$ to $20.4 \%$ over a period of 7 days (Fig. 3). Patients with stage 1 acute kidney injury on day 1 were more likely to have progression to stage 2 or 3 by day 7 than patients without acute kidney injury on day $1(14.1 \%$ vs. $2.9 \%, \mathrm{P}<0.001)$. 


\section{Predictors of Severe Acute Kidney Injury}

Several factors were associated with severe acute kidney injury (Table S6 in the Supplementary Appendix). Transplantation was associated with the highest risk (odds ratio, $2.43 ; 95 \% \mathrm{CI}, 1.71$ to 3.40 ). The distribution of severe acute kidney injury was similar in patients who had undergone solid organ transplantation and those who had undergone bone marrow transplantation (Table S7 in the Supplementary Appendix).

\section{Center Variability}

Characteristics of the ICUs are shown in Table S8 in the Supplementary Appendix. We observed in-tercenter variability in the rate of severe acute kidney injury (median, 10.4\%; interquartile range, 7.8 to 16.7) and in the rate of death (median, 3.2\%; interquartile range, 2.0 to 6.1 ). The rates for each individual center and the rates pooled according to country are shown in Figures S2 and S3 in the Supplementary Appendix.

\section{Discussion}

This large, prospective, multinational study of the epidemiology of acute kidney injury in children and young adults in ICUs showed that acute kidney injury occurred in one quarter of patients during the first 7 days after ICU admission. Severe acute kidney injury conferred an incremental risk of death by day 28 and was associated with increased use of renalreplacement therapy and mechanical ventilation and longer stays in the ICU. Assessment of acute kidney injury according to the plasma creatinine level alone failed to identify acute kidney injury in two thirds of the patients with low urine output, and low urine output alone conferred an increased risk of death.

The presence of chronic systemic diseases contributes to residual confounding in studies of acute kidney injury in adults. Children have a low prevalence of such chronic diseases; thus, although the incremental association between acute kidney injury and risk of death mirrors that seen in adults, our study suggests that acute kidney injury itself may be key to the associated morbidity and mortality.

The common and early occurrence of acute kidney injury reinforces the need for systematic surveillance for acute kidney injury at the time of admission to the ICU. Early identification of modifiable risk factors for acute kidney injury (e.g., nephrotoxic medications) or adverse sequelae (e.g., fluid overload) has the potential to decrease morbidity and mortality. ${ }^{19-21}$ Previously reported rates of acute kidney injury among children and young adults vary widely - from 5\% to $82 \% .6,7,11,12,22$ This variation probably results from differences in case mix, illness severity, coexisting conditions, and definitions of acute kidney injury. A 5\% rate of acute kidney injury was reported in a single-center study that used a $100 \%$ rise in the plasma creatinine level to define acute kidney injury, ${ }^{22}$ whereas in another study, $82 \%$ of patients receiving at least one vasoactive medication and mechanical ventilation had acute kidney injury as defined by a $33 \%$ rise in the plasma creatinine level. ${ }^{6}$ Since the adoption of standardized classification criteria for acute kidney injury, reported rates among critically ill children have ranged from 10 to $40 \% .6,7,10-12$ Although we observed intercenter variation in rates of severe acute kidney injury and death, the $95 \%$ confidence intervals overlap for the 
vast majority of sites and countries. The pooled $26.9 \%$ rate of acute kidney injury in the current study may therefore be regarded as a valid benchmark, given the large sample and multinational composition.

Our study results provide informative comparisons with those of a recent multicenter study in adults, the Acute Kidney Injury-Epidemiologic Prospective Investigation (AKI-EPI) study. ${ }^{3}$ Although the rates of overall and severe acute kidney injury (57.3\% and $38.9 \%$, respectively) were higher in the adult study than the corresponding rates in our study, the associations between acute kidney injury and mortality and morbidity are similar. ${ }^{3} \mathrm{We}$ speculate that the relatively lower rates of acute kidney injury observed in our study represent greater renal reserve in children. Although both studies show an increased risk of death with higher KDIGO stages (odds ratios ranged from 1.18 at stage 1 to 8.21 at stage 3 in our study and from 2.19 at stage 1 to 7.18 at stage 3 in the AKI-EPI study), our study did not show an association between stage 1 acute kidney injury and mortality. The multiple coexisting conditions seen in adults may confer greater susceptibility to acute kidney injuryassociated outcomes, which is, in part, supported by the higher overall mortality seen in the AKI-EPI study than in our study (18.4\% vs. $3.4 \%)$. The current study reinforces the importance of using both the plasma creatinine level and urine output to define acute kidney injury. ${ }^{5}$ This finding questions nationwide efforts to prevent urinary tract infections by not placing indwelling bladder catheters, or removing them early, in all critically ill children. ${ }^{23}$ Our data suggest that children are more likely to survive severe acute kidney injury than adults. Because children who survive acute kidney injury are at risk for chronic kidney disease, long-term follow-up of these survivors is warranted. ${ }^{24,25}$

Although the daily prevalence of acute kidney injury increased from $14.5 \%$ on day 1 to $20.4 \%$ on day 7 , approximately $75 \%$ of the increase occurred within 4 days after admission. This increase probably results from the earlier discharge of less severely ill patients who would be at lower risk for multiorgan failure and acute kidney injury. Such data may support the use of a 4-day time frame for future focused studies of acute kidney injury in children.

Our study has a number of strengths. It involved a large, multicenter cohort of children and young adults, which enabled a robust evaluation of relationships between exposure and outcome, and it had a prespecified protocol, operational definitions, and an analysis plan and enumerated complete standardized diagnostic criteria for acute kidney injury. The overall mortality of $3.4 \%$ is similar to the rate reported in epidemiologic studies conducted in pediatric ICUs, ${ }^{26,27}$ which suggests that our cohort is representative.

The study also has some limitations. First, because it is an observational cohort study, we cannot make statements regarding causal relationships among acute kidney injury, exposures, and outcomes observed. Second, we cannot generalize our findings outside pediatric ICU settings. Third, we did not assess for the potential effect of the specific cause of acute kidney injury on patient outcomes. Fourth, we have no detailed information on interventions (e.g., use of diuretics) that could increase urinary output and thereby alter the observed prevalence of acute kidney injury. Fifth, we required one plasma creatinine measurement or 12 hours of recorded urine output in the ICU to constitute sufficient data for assessment of acute kidney injury. Although more data could alter the perceived rates, $94 \%$ 
of patients with data on 28-day outcomes met the assessment requirements for the plasma creatinine level or urine output. Sixth, assessment for acute kidney injury stopped at day 7 in our study, and it is possible that we missed events of acute kidney injury occurring later. Seventh, secondary outcomes (e.g., provision of renal-replacement therapy or mechanical ventilation) were driven by local care practice and therefore were potentially biased by pediatric inten-sivists who were interested in acute kidney injury. Finally, although the current study represents a large international pediatric study, two thirds of the centers were from North America; we were unable to recruit centers from Africa and South America.

In conclusion, our study involving critically ill children and young adults showed that acute kidney injury is common and is associated with poor outcomes, including increased mortality.

\section{Supplementary Material}

Refer to Web version on PubMed Central for supplementary material.

\section{Acknowledgments}

Supported by a grant (NIH P50 DK096418, to Drs. Basu and Goldstein) from the Pediatric Nephrology Center of Excellence at Cincinnati Children's Hospital Medical Center. Dr. Kaddourah's Pediatric Acute Care Nephrology and Dialysis Fellowship at Cincinnati Children's Hospital Medical Center was supported by an educational grant from Gambro Renal Products.

We thank the Cincinnati Children's Research Foundation, successively headed by Arnold Strauss, M.D., and Margaret Hostetter, M.D., during the course of the study, for waiving all fees for development of the Medidata Rave AWARE database.

\section{References}

1. Uchino S, Kellum JA, Bellomo R, et al. Acute renal failure in critically ill patients: a multinational, multicenter study. JAMA. 2005; 294:813-818. [PubMed: 16106006]

2. Chertow GM, Burdick E, Honour M, Bonventre JV, Bates DW. Acute kidney injury, mortality, length of stay, and costs in hospitalized patients. J Am Soc Nephrol. 2005; 16:3365-3370. [PubMed: 16177006]

3. Hoste EA, Bagshaw SM, Bellomo R, et al. Epidemiology of acute kidney injury in critically ill patients: the multinational AKI-EPI study. Intensive Care Med. 2015; 41:1411-1423. [PubMed: 26162677]

4. Kidney Disease: Improving Global Outcomes (KDIGO) Acute Kidney Injury Work Group. KDIGO clinical practice guideline for acute kidney injury. Kidney Int. 2012; 2(Suppl 1):1-138.

5. Kellum JA, Sileanu FE, Murugan R, Lucko N, Shaw AD, Clermont G. Classifying AKI by urine output versus serum cre-atinine level. J Am Soc Nephrol. 2015; 26:2231-2238. [PubMed: 25568178]

6. Akcan-Arikan A, Zappitelli M, Loftis LL, Washburn KK, Jefferson LS, Goldstein SL. Modified RIFLE criteria in critically ill children with acute kidney injury. Kidney I nt. 2007; 71:1028-1035.

7. Schneider J, Khemani R, Grushkin C, Bart R. Serum creatinine as stratified in the RIFLE score for acute kidney injury is associated with mortality and length of stay for children in the pediatric intensive care unit. Crit Care Med. 2010; 38:933-939. [PubMed: 20124891]

8. Sutherland SM, Ji J, Sheikhi FH, et al. AKI in hospitalized children: epidemiology and clinical associations in a national cohort. Clin J Am Soc Nephrol. 2013; 8:1661-169. [PubMed: 23833312]

9. Sutherland SM, Goldstein SL, Alexander SR. The Prospective Pediatric Continuous Renal Replacement Therapy (ppCRRT) Registry: a critical appraisal. Pediatr Nephrol. 2014; 29:20692076. [PubMed: 23982708] 
10. Selewski DT, Cornell TT, Heung M, et al. Validation of the KDIGO acute kidney injury criteria in a pediatric critical care population. Intensive Care Med. 2014; 40:1481-1488. [PubMed: 25079008]

11. Sutherland SM, Byrnes JJ, Kothari M, et al. AKI in hospitalized children: comparing the pRIFLE, AKIN, and KDIGO definitions. Clin J Am Soc Nephrol. 2015; 10:554-561. [PubMed: 25649155]

12. Sanchez-Pinto LN, Goldstein SL, Schneider JB, Khemani RG. Association between progression and improvement of acute kidney injury and mortality in critically ill children. Pediatr Crit Care Med. 2015; 16:703-10. [PubMed: 26132741]

13. Lex DJ, Tóth R, Cserép Z, et al. A comparison of the systems for the identification of postoperative acute kidney injury in pediatric cardiac patients. Ann Thorac Surg. 2014; 97:202-210. [PubMed: 24206964]

14. Basu RK, Kaddourah A, Terrell T, et al. Assessment of Worldwide Acute Kidney Injury, Renal Angina and Epidemiology in critically ill children (AWARE): study protocol for a prospective observational study. BMC Nephrol. 2015; 16:24. [PubMed: 25882434]

15. Schwartz GJ, Haycock GB, Edelmann CM Jr, Spitzer A. A simple estimate of glomerular filtration rate in children derived from body length and plasma creatinine. Pediatrics. 1976; 58:259-263. [PubMed: 951142]

16. Zappitelli M, Parikh CR, Akcan-Arikan A, Washburn KK, Moffett BS, Goldstein SL. Ascertainment and epidemiology of acute kidney injury varies with definition interpretation. Clin J Am Soc Nephrol. 2008; 3:948-954. [PubMed: 18417742]

17. Slater MB, Anand V, Uleryk EM, Parshuram CS. A systematic review of RIFLE criteria in children, and its application and association with measures of mortality and morbidity. Kidney Int. 2012; 81:791-798. [PubMed: 22258324]

18. Basu RK, Chawla LS, Wheeler DS, Goldstein SL. Renal angina: an emerging paradigm to identify children at risk for acute kidney injury. Pediatr Nephrol. 2012; 27:1067-1078. [PubMed: 22012033]

19. Menon S, Kirkendall ES, Nguyen H, Goldstein SL. Acute kidney injury associated with high nephrotoxic medication exposure leads to chronic kidney disease after 6 months. J Pediatr. 2014; 165(3):522-7.e2. [PubMed: 24928698]

20. Sutherland SM, Zappitelli M, Alexander SR, et al. Fluid overload and mortality in children receiving continuous renal replacement therapy: the Prospective Pediatric Continuous Renal Replacement Therapy Registry. Am J Kidney Dis. 2010; 55:316-325. [PubMed: 20042260]

21. Arikan AA, Zappitelli M, Goldstein SL, Naipaul A, Jefferson LS, Loftis LL. Fluid overload is associated with impaired oxy-genation and morbidity in critically ill children. Pediatr Crit Care Med. 2012; 13:253-258. [PubMed: 21760565]

22. Bailey D, Phan V, Litalien C, et al. Risk factors of acute renal failure in critically ill children: a prospective descriptive epi-demiological study. Pediatr Crit Care Med. 2007; 8:29-35. [PubMed: 17251879]

23. Davis KF, Colebaugh AM, Eithun BL, et al. Reducing catheter-associated urinary tract infections: a quality-improvement initiative. Pediatrics. 2014; 134(3):e857-e864. [PubMed: 25113293]

24. Goldstein SL, Jaber BL, Faubel S, Chawla LS. AKI transition of care: a potential opportunity to detect and prevent CKD. Clin J Am Soc Nephrol. 2013; 8:476-483. [PubMed: 23471414]

25. Silver SA, Goldstein SL, Harel Z, et al. Ambulatory care after acute kidney injury: an opportunity to improve patient outcomes. Can J Kidney Health Dis. 2015; 2:36. [PubMed: 26445676]

26. Pollack MM, Holubkov R, Funai T, et al. The Pediatric Risk of Mortality score: update 2015. Pediatr Crit Care Med. 2016; 17:2-9. [PubMed: 26492059]

27. Markovitz BP, Kukuyeva I, Soto-Campos G, Khemani RG. PICU volume and outcome: a severityadjusted analysis. Pediatr Crit Care Med. 2016; 17:483-489. [PubMed: 26959348] 


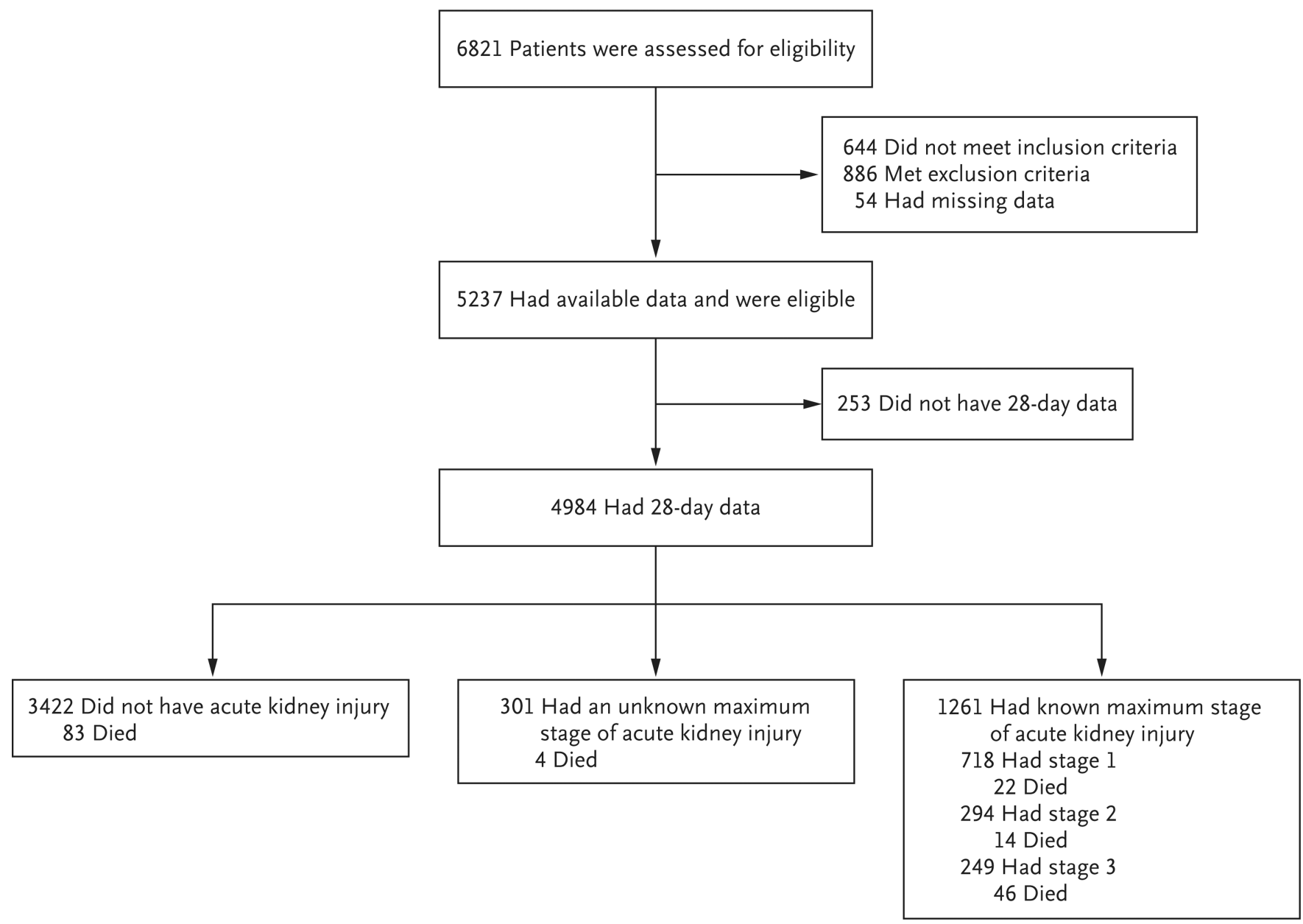

Figure 1. Screening, Eligibility, and 28-Day Primary Outcome

The primary outcome was death by day 28 after admission to the pediatric intensive care unit (ICU). The 253 patients who did not have 28 -day data were excluded from outcome analyses. The maximum stage of acute kidney injury was defined as the highest stage observed during the first 7 days after ICU admission. The stages of acute kidney injury were defined according to the Kidney Disease: Improving Global Outcomes plasma creatinine criteria (stage 1: an increase in the creatinine level to 1.5 to 1.9 times the baseline level that is known or presumed to have occurred within the past 7 days or an increase by $\searrow 0.3 \mathrm{mg}$ per deciliter [26.5 $\mu \mathrm{mol}$ per liter] within the past 48 hours; stage 2: a level 2.0 to 2.9 times the baseline level; stage 3: a level 3.0 times the baseline level, an increase to $\geq 4.0 \mathrm{mg}$ per deciliter [354 $\mu \mathrm{mol}$ per liter], or, in patients <18 years of age, a decrease in the estimated glomerular filtration rate to $<35 \mathrm{ml}$ per minute per $1.73 \mathrm{~m}^{2}$ ) and urine-output criteria (stage 1: $<0.5 \mathrm{ml}$ per kilogram of body weight per hour for 6 to $<12$ hours; stage $2:<0.5 \mathrm{ml}$ per kilogram per hour for $\geq 12$ hours; stage $3:<0.3 \mathrm{ml}$ per kilogram per hour for $\geq 24$ hours or anuria for $\geq 12$ hours). 
$P$ Value

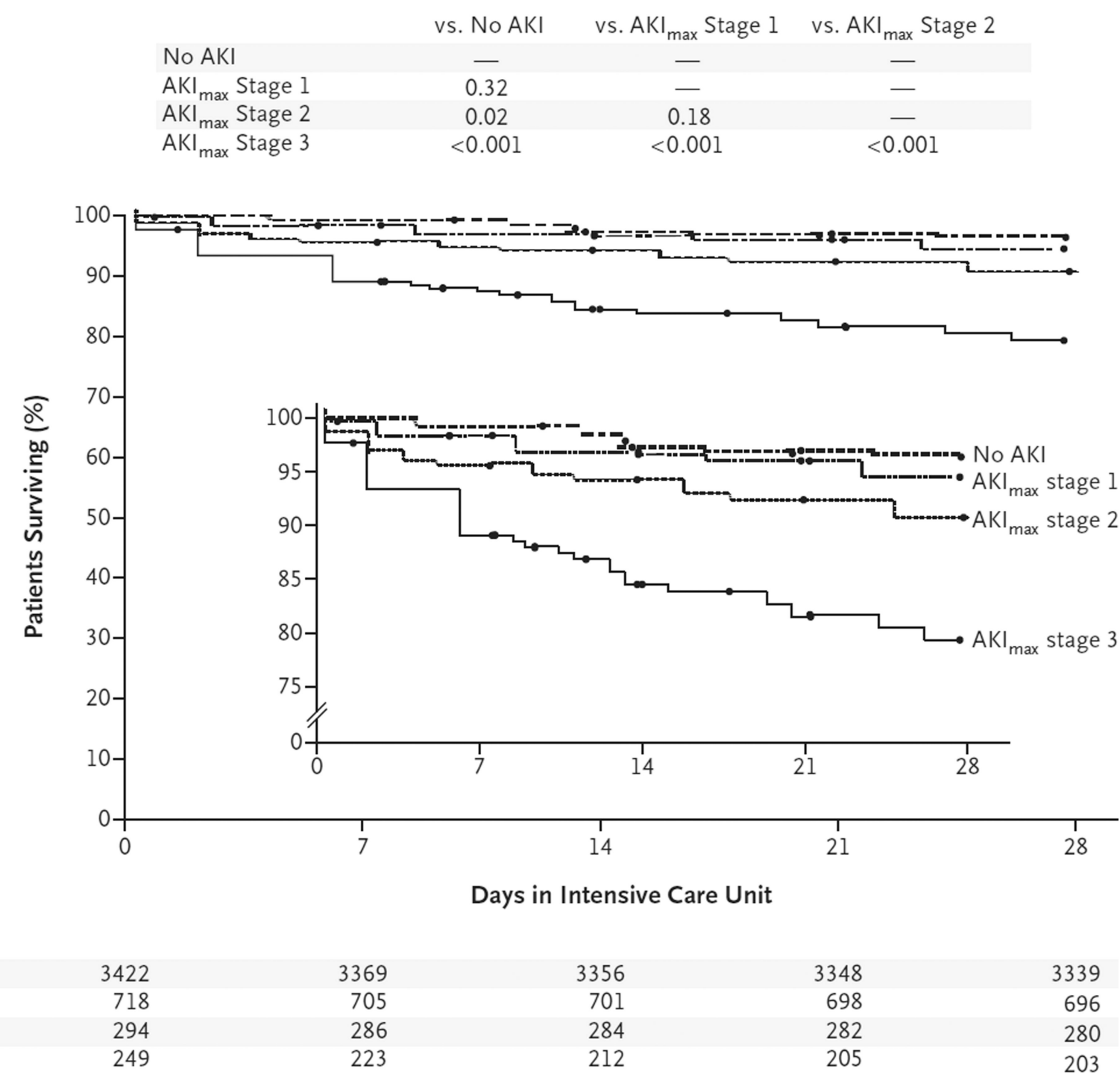

Figure 2. Kaplan-Meier Survival Curves According to Maximum Stage of Acute Kidney Injury $\left(\mathbf{A K I} \mathbf{m a x}_{\max }\right)$

Death occurred in four patients in whom $\mathrm{AKI}_{\max }$ was unknown; these patients were excluded from the analysis. The inset shows the same data on an expanded y axis. 


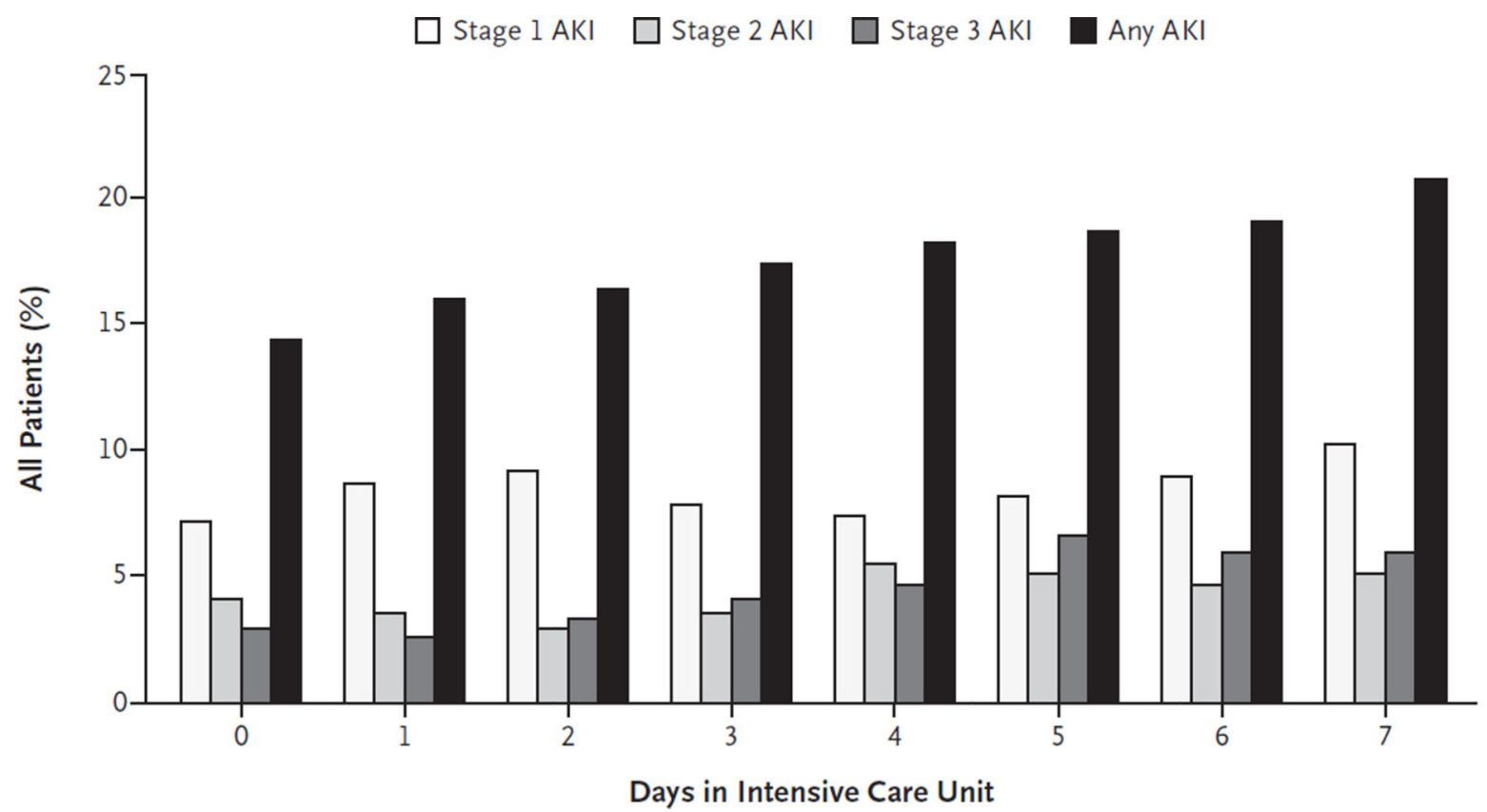

All Patients (\%)

\begin{tabular}{|c|c|c|c|c|c|c|c|c|}
\hline Stage 1 AKI & 7.3 & 9.7 & 9.8 & 9.0 & 8.6 & 9.0 & 9.8 & 10.4 \\
\hline Stage $2 \mathrm{AKI}$ & 4.1 & 3.9 & 3.5 & 4.2 & 5.4 & 4.4 & 4.3 & 4.7 \\
\hline Stage 3 AKI & 3.1 & 2.6 & 3.6 & 4.4 & 4.9 & 5.7 & 5.3 & 5.3 \\
\hline Any AKI & 14.5 & 16.2 & 16.8 & 17.5 & 18.9 & 19.1 & 19.4 & 20.4 \\
\hline
\end{tabular}

Figure 3. Prevalence of Acute Kidney Injury, According to Stage, during the First Week of ICU Admission

For day 0 , only the serum creatinine level was used to assess for acute kidney injury because data on urine output were not available. Patients with missing data to assess for acute kidney injury were excluded from analysis. 


\section{Table 1}

Baseline Characteristics of the Study Cohort. *

\begin{tabular}{|c|c|}
\hline Variable & Value \\
\hline Patients — no. & 5237 \\
\hline Male sex — no. $(\%)$ & $2878(55.0)$ \\
\hline \multicolumn{2}{|l|}{ Race or ethnic group — no. $(\%)^{\dagger}$} \\
\hline White & $3120(59.6)$ \\
\hline Black & $883(16.9)$ \\
\hline Native American & $61(1.2)$ \\
\hline Asian & $480(9.2)$ \\
\hline Hawaiian or Pacific Islander & $27(0.5)$ \\
\hline Other or unknown & $666(12.7)$ \\
\hline \multicolumn{2}{|l|}{ Age - mo } \\
\hline Median & 66.0 \\
\hline IQR & $18.8-151.1$ \\
\hline Height at ICU admission $-\mathrm{cm}$ & $114.8 \pm 37.7$ \\
\hline \multicolumn{2}{|l|}{ Body-surface area $-\mathrm{m}^{2}$} \\
\hline Median & 0.77 \\
\hline IQR & $0.49-1.30$ \\
\hline $\begin{array}{l}\text { Patients with a measured baseline } \\
\text { plasma creatinine level } \\
\text { - no. }(\%)\end{array}$ & $2539(48.5)$ \\
\hline \multicolumn{2}{|l|}{ Estimated GFR $-\mathrm{ml} / \mathrm{min} / 1.73 \mathrm{~m}^{2 / \hbar}$} \\
\hline \multicolumn{2}{|l|}{ All patients } \\
\hline Median & 120.0 \\
\hline IQR & $120.0-165.6$ \\
\hline \multicolumn{2}{|l|}{$\begin{array}{l}\text { Patients with a measured base- } \\
\text { line plasma creatinine } \\
\text { level }\end{array}$} \\
\hline Median & 168.7 \\
\hline IQR & $120.5-224.6$ \\
\hline \multicolumn{2}{|c|}{$\begin{array}{l}\text { Primary diagnosis group at ICU admission } \\
- \text { no. }(\%)^{\xi}\end{array}$} \\
\hline Shock & $1244(23.8)$ \\
\hline Cardiovascular & $210(4.0)$ \\
\hline Respiratory & $1986(37.9)$ \\
\hline Surgical or trauma & $1597(30.5)$ \\
\hline Central nervous system & $958(18.3)$ \\
\hline Pain management or sedation & $182(3.5)$ \\
\hline \multicolumn{2}{|l|}{ Coexisting condition - no. $(\%)^{\mathcal{S}}$} \\
\hline Cardiovascular & $658(12.6)$ \\
\hline
\end{tabular}




\begin{tabular}{|c|c|}
\hline Variable & Value \\
\hline Pulmonary & $1922(36.7)$ \\
\hline Neurologic & $1846(35.2)$ \\
\hline Gastrointestinal & $925(17.7)$ \\
\hline Renal or urologic & $329(6.3)$ \\
\hline Hematologic & $356(6.8)$ \\
\hline Oncologic & $399(7.6)$ \\
\hline Immunologic & $124(2.4)$ \\
\hline Infectious disease & $376(7.2)$ \\
\hline Rheumatologic & $62(1.2)$ \\
\hline Neuromuscular & $702(13.4)$ \\
\hline Metabolic & $575(11.0)$ \\
\hline \multicolumn{2}{|l|}{ History of transplantation } \\
\hline Any — no. (\%) & $219(4.2)$ \\
\hline \multicolumn{2}{|l|}{$\begin{array}{l}\text { Type of transplantation } \\
- \text { no./total no. (\%) }\end{array}$} \\
\hline Hematopoietic stem cell & $93 / 219(42.5)$ \\
\hline Liver & $82 / 219(37.4)$ \\
\hline Kidney & $13 / 219(5.9)$ \\
\hline Small bowel & $5 / 219(2.3)$ \\
\hline Pancreas & $1 / 219(0.5)$ \\
\hline Multivisceral & $2 / 219(0.9)$ \\
\hline Heart & $20 / 219(9.1)$ \\
\hline Lung & $12 / 219(5.5)$ \\
\hline
\end{tabular}

* Plus-minus values are means \pm SD. ICU denotes intensive care unit, and IQR interquartile range.

Race and ethnic group were reported by patients 18 years of age or older who were physically able to provide the information or by parents or guardians if the patient was younger than 18 years of age or too critically ill to provide the information.

t

The estimated glomerular filtration rate (GFR) was calculated with the original Schwartz formula, in which estimated GFR $=\mathrm{k} \times$ patient height (in centimeters) $\div$ plasma creatinine (in milligrams per deciliter); $\mathrm{k}$ is a constant defined as 0.45 (infant younger than 1 year of age), 0.55 (child or female adolescent), or 0.70 (male adolescent). ${ }^{19}$

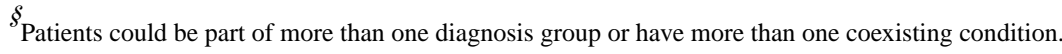




\section{Table 2}

Bivariate and Multivariable Logistic-Regression Analysis of Predictors of Death by Day 28 after Admission.

\begin{tabular}{|c|c|c|c|c|}
\hline \multirow[b]{2}{*}{ Variable } & \multicolumn{3}{|c|}{ Bivariate Analysis } & \multirow{2}{*}{$\begin{array}{l}\text { Multivariable Logistic- } \\
\text { Regression Analysis } \\
\text { Odds Ratio (95\% CI) }\end{array}$} \\
\hline & $\begin{array}{l}\text { Survivors } \\
(\mathrm{N}=\mathbf{4 8 1 5})\end{array}$ & $\begin{array}{c}\text { Nonsurvivors } \\
(\mathbf{N}=169)\end{array}$ & P Value & \\
\hline \multicolumn{5}{|l|}{ Primary diagnosis group at ICU admission - no. $(\%)^{\dagger}$} \\
\hline Shock & $1101(22.9)$ & $92(54.4)$ & $<0.001$ & $1.93(1.31-2.84)$ \\
\hline Cardiovascular & $178(3.7)$ & $28(16.6)$ & $<0.001$ & $2.71(1.50-4.89)$ \\
\hline Respiratory & $1814(37.7)$ & $87(51.5)$ & 0.003 & $1.27(0.86-1.86)$ \\
\hline Surgical or trauma & $1481(30.8)$ & $12(7.1)$ & $<0.001$ & $0.42(0.22-0.80)$ \\
\hline Central nervous system & $873(18.1)$ & $46(27.2)$ & 0.003 & $2.42(1.60-3.68)$ \\
\hline Pain management or sedation & $164(3.4)$ & $2(1.2)$ & 0.06 & $0.33(0.07-1.54)$ \\
\hline \multicolumn{5}{|l|}{ Coexisting condition at baseline - no. $(\%)^{\dagger}$} \\
\hline Cardiovascular & $607(12.6)$ & $40(23.7)$ & $<0.001$ & $1.20(0.75-1.91)$ \\
\hline Pulmonary & $1785(37.1)$ & $60(35.5)$ & 0.68 & - \\
\hline Neurologic & $1699(35.3)$ & $57(33.7)$ & 0.68 & - \\
\hline Gastrointestinal & $875(18.2)$ & $32(18.9)$ & 0.80 & - \\
\hline Renal or urologic & $307(6.4)$ & $14(8.3)$ & 0.34 & - \\
\hline Hematologic & $307(6.4)$ & $35(20.7)$ & $<0.001$ & $2.99(1.86-4.82)$ \\
\hline Oncologic & $353(7.3)$ & $23(13.6)$ & 0.01 & $1.70(0.97-2.90)$ \\
\hline Immunologic & $101(2.1)$ & $8(4.7)$ & 0.04 & $1.31(0.54-3.18)$ \\
\hline Infectious disease & $351(7.3)$ & $25(14.8)$ & 0.006 & $1.06(0.60-1.80)$ \\
\hline Rheumatologic & $61(1.3)$ & $1(0.6)$ & 0.38 & - \\
\hline Neuromuscular & $601(12.5)$ & $21(12.4)$ & 0.98 & - \\
\hline Metabolic & $539(11.2)$ & $22(13.0)$ & 0.16 & - \\
\hline History of transplantation - no. $(\%)$ & $195(4.0)$ & $15(8.9)$ & 0.007 & $0.86(0.43-1.70)$ \\
\hline \multicolumn{5}{|l|}{ Estimated GFR $-\mathrm{ml} / \mathrm{min} / 1.73 \mathrm{~m}^{2}$} \\
\hline Median & 120 & 120 & 0.95 & - \\
\hline $\mathrm{IQR}$ & $120-166$ & $120-181$ & & \\
\hline Maximum stage of acute kidney injury - no./total no. $(\%)^{f t}$ & & & $<0.001$ & \\
\hline No acute kidney injury & $3339 / 4518(73.9)$ & $83 / 165(50.3)$ & & - \\
\hline Stage 1 & $696 / 4518(15.4)$ & $22 / 165(13.3)$ & & - \\
\hline Stage 2 & $280 / 4518(6.2)$ & $14 / 165(8.5)$ & & - \\
\hline Stage 3 & $203 / 4518(4.5)$ & $46 / 165(27.9)$ & & - \\
\hline Stage 2 or 3 : severe acute kidney injury & $483 / 4518(10.7)$ & $60 / 165(36.4)$ & $<0.001$ & $1.77(1.17-2.68)$ \\
\hline Ventricular assist device - no. $(\%)$ & $7(0.1)$ & 0 & 0.49 & - \\
\hline Extracorporeal membrane oxygenation — no. (\%) & $20(0.4)$ & $6(3.6)$ & 0.002 & $1.12(0.37-3.39)$ \\
\hline Renal-replacement therapy — no. (\%) & $49(1.0)$ & $24(14.2)$ & $<0.001$ & $3.38(1.74-6.54)$ \\
\hline Mechanical ventilation — no. $(\%)$ & $1456(30.2)$ & $125(74.0)$ & $<0.001$ & $3.02(2.16-4.76)$ \\
\hline
\end{tabular}

N Engl J Med. Author manuscript; available in PMC 2017 July 05. 


\begin{tabular}{|c|c|c|c|c|}
\hline \multirow[b]{2}{*}{ Variable } & \multicolumn{3}{|c|}{ Bivariate Analysis } & \multirow{2}{*}{$\begin{array}{l}\text { Multivariable Logistic- } \\
\text { Regression Analysis } \\
\text { Odds Ratio }(95 \% \mathrm{CI})\end{array}$} \\
\hline & $\begin{array}{l}\text { Survivors } \\
(N=4815)\end{array}$ & $\begin{array}{c}\text { Nonsurvivors } \\
(\mathbf{N}=169)\end{array}$ & P Value & \\
\hline Vasoactive support - no. $(\%)$ & $618(12.8)$ & $108(63.9)$ & $<0.001$ & $4.67(3.18-6.87)$ \\
\hline
\end{tabular}

The reference for the odds ratio is the absence of the corresponding risk factor. Variables were entered into the multivariable logistic-regression model (and, therefore, odds ratios were calculated) when the alpha level of the risk factor was less than 0.15 in bivariate analysis.

\section{t}

${ }^{t}$ Patients could be part of more than one diagnosis group or have more than one coexisting condition.

${ }^{*}$ The analysis was limited to patients with known status with respect to both acute kidney injury and 28-day outcome (alive vs. dead). The stages of acute kidney injury were defined according to the Kidney Disease: Improving Global Outcomes plasma creatinine criteria (stage 1: an increase in the creatinine level to 1.5 to 1.9 times the baseline level that is known or presumed to have occurred within the past 7 days or an increase by $\searrow .3$ mg per deciliter [ $26.5 \mu \mathrm{mol}$ per liter] within the past 48 hours; stage 2: a level 2.0 to 2.9 times the baseline level; stage 3: a level 3.0 times the baseline level, an increase to $24.0 \mathrm{mg}$ per deciliter [ $354 \mu \mathrm{mol}$ per liter], or, in patients $<18$ years of age, a decrease in the estimated GFR to $<35 \mathrm{ml}$ per minute per $1.73 \mathrm{~m}^{2}$ ) and urine-output criteria (stage 1: $<0.5 \mathrm{ml}$ per kilogram of body weight per hour for 6 to $<12$ hours; stage $2:<0.5 \mathrm{ml}$ per kilogram per hour for $\geq 12$ hours; stage 3: $<0.3 \mathrm{ml}$ per kilogram per hour for $\geq 24$ hours or anuria for $\geq 12$ hours). The 16 covariates assessed for the multivariable logistic-regression model were a diagnosis at ICU admission of shock, cardiovascular disease, respiratory disease, surgical or trauma, central nervous system disorder, or pain or sedation; the presence of cardiovascular, hematologic, oncologic, immunologic, or infectious conditions at baseline; a history of transplantation; and the use of extracorporeal membrane oxygenation, renal-replacement therapy, mechanical ventilation, or vasoactive support. 